\title{
Generalized or Adjoint Reciprocity Relations for Electroacoustic Transducers*
}

\author{
A. D. Yaghiian \\ Institute for Basic Standards, National Bureau of Standards, Boulder, Colorado 80302
}

(November 4, 1974)

\begin{abstract}
The "equations of motion" of a linear electroacoustic transducer are written in the alternative forms $L+X=0, L-X=0$, where the matrices $L^{ \pm}$are linear differential expressions, $X=(\bar{E}, \bar{H}, \bar{u})$ represents electroacoustic fields, and the superscripts distinguish selected normalizations of the equations of motion. To each operator $L^{ \pm}$corresponds a mathematically defined adjoint operator $\left(L^{ \pm}\right)^{a}$ and an associated adjoint transducer, whose material tensor parameters are given by certain transpositions and interchanges of the parameters of the given transducer. Dissipative characteristics (lossiness, losslessness, or "gaininess") of the material of the given transducer are preserved pointwise in the adjoint transducers. A generalized reciprocity lemma leads to relations of reciprocal type between external properties of the given and the adjoint transducers. In the self-adjoint cases, the conventional electroacoustic reciprocity and antireciprocity relations are obtained and the derivation of those relations is critically confirmed. The generalized or adjoint reciprocity relations have been applied in the plane-wave scattering-matrix formalism developed for electroacoustic transducers by D. M. Kerns.

Corollaries of the adjoint reciprocity relations, analogous to conventional reciprocity theorems, but involving properties of adjoint pairs of transducers, are derived. Examples are discussed of transducers for which it is feasible to form the adjoint transducers.
\end{abstract}

Key words: Linear differential operators; reciprocity relations; scattering matrix; transducers.

\section{Glossary of Symbols}

$a$-superscript: Denotes the adjoint.

$t$-subscript:

Denotes the transpose.

$A_{0}, A_{p}, A$ :

Surface of transducer; surface of power supply or detector; and surface of transducer-plus-(power supply or detector).

$a_{0}, b_{0}$ : Amplitude of incident and emergent em mode which feeds the transducer.

$a(\bar{K}), b(\bar{K})$ : Incoming and outgoing plane-wave amplitudes in ambient fluid.

$\overline{\bar{\alpha}}, \overline{\bar{\alpha}}^{\prime}$ : $\quad$ Direct and converse piezoelectric stress tensor (triadic).

$\mathscr{A}:$

Amplitude of spherical wave in ambient fluid.

$\bar{B}, \bar{B}_{0}$ :

Harmonic and static magnetic induction.

$\overline{\bar{\beta}}, \overline{\bar{\beta}}^{\prime}$ :

Direct and converse piezomagnetic stress tensor (triadic).

$\overline{\bar{c}}$ :

Hooke's tensor (tetradic).

$\bar{D}$ :

Electric displacement.

$\bar{E}, \bar{E}_{0}$ :

Harmonic and static electric field.

$\bar{e}_{0}, \bar{h}_{0}$ :

Electric and magnetic field components of em mode which feeds the transducer.

AMS Subject Classification: 7000, 7800.

*This work was done while the author was a National Research Council Postdoctoral Associate in the Electromagnetics Division of the National Bureau of Standards, Boulder, Colorado. 
$\bar{\epsilon}$ :

$\eta=\gamma / \omega \rho_{0}:$

$\eta_{0}$ :

$\bar{F}$ :

$\bar{f}, \bar{f}_{s}:$

$\gamma:$

$\bar{H}$ :

$h, h^{\prime}$ :

$\overline{\bar{I}}$ :

$\bar{J}, \bar{J}_{s}:$

$\bar{K}, \bar{L}$ :

$\bar{k}, \bar{l}$ :

$L, L^{-}$:

$\bar{M}_{s}$ :

$M(\bar{r})$ :

$\bar{\mu}$ :

$\mu_{0}$ :

$p, p_{0}$ :

$\overline{\mathrm{P}}_{s}$ :

$\rho_{0}$ :

$\rho_{m}, \rho_{m}^{\circ}, \rho_{m}^{\prime}$ :

$\rho_{e}, \rho_{e}^{\circ}$ :

$S_{0}$ :

$S_{1}$ :

$S_{00}, S_{01}, S_{10}, S_{11}$ :

$\mathrm{S}(\bar{r})$ :

$\bar{s}$ :

$\overline{\bar{\sigma}}:$

$\overline{\bar{T}}, \overline{\bar{T}}_{0}, \overline{\bar{T}}^{\prime}$ :

$\overline{\bar{\tau}}, \overline{\bar{\nu}}$ :

$\bar{u}$ :

$V_{0}, I_{0}$ :

$V(\bar{K}), I(\bar{K})$ :

$Z_{b}, Z_{0}$ :

Permittivity dyadic.

Plane-wave admittance.

Admittance of em mode feeding transducer.

Bilinear concomitant associated with the operator $L$.

Induced and applied volume forces.

$z$-component of the plane-wave propagation vector.

Magnetic field.

Spacial impedances describing transducer.

Identity dyadic.

Induced and applied current densities.

Transverse part of $\bar{k}$ and $\bar{l}$.

Plane-wave propagation vectors in ambient fluid.

Transducer operator matrices.

Applied magnetization.

Microphone response.

Permeability dyadic.

Free-space permeability.

Harmonic and static pressure.

Applied polarization.

Static mass density of the ambient fluid.

Harmonic, static, and total mass density of transducer material.

Harmonic and static charge density.

Transducer feed surface.

Reference $x y$ plane in ambient fluid.

Plane-wave scattering matrices describing transducer.

Speaker response.

Strain dyadic.

Conductivity dyadic.

Harmonic, static, and total stress dyadic.

Magneto-electric dyadics.

Fluid or material velocity.

Input voltage and current to transducer.

Voltage and current spectral densities.

Spacial impedances (along with $h, h^{\prime}$ ) describing transducer.

\section{Introduction}

Analytical techniques developed by Kerns $[1,2,3]^{1}$ for the measurement of microwave antennas have been "translated" recently by that author into corresponding techniques for the measurement of electroacoustic transducers in fluids. The basic theory is formulated with a scatteringmatrix description and emphasizes the use of plane-wave spectra for the representation of acoustic fields. For a summary and for details of the theory and its variety of applications, reference should be made to Kerns [4].

Although the electromagnetic (em) theory is quite comprehensive, the acoustics applications are strengthened by generalized or adjoint reciprocity relations [5], the derivation of which forms the principal subject of the present paper. The generalized reciprocity relations are expressed in the notation of the Kerns plane-wave scattering-matrix (PWSM) description, and also in the notation of the spacial impedance-matrix $(S I M)$ description introduced by Foldy and Primakoff $[6,7]$ in their classic papers on electroacoustic transducers and reciprocity. The PWSM expres-

${ }^{1}$ Figures in brackets indicate the literature references at the end of this paper 
sions of adjoint reciprocity have become especially important because of their use in the theoretical foundation of extrapolation techniques which predict gain on an arbitrary axis from measurements at reduced distances along that axis [4]. (Extrapolation techniques were introduced for antennas by Wacker and Bowman [8].)

The following approach is taken to establish the adjoint reciprocity relationships. The equations of motion and constitutive relations describing a large class of linear electroacoustic transducers are reduced to the form of a linear operator matrix. The operator is "transposed" to form a unique adjoint operator, which is compared and combined with the original operator to derive a generalized electroacoustic reciprocity lemma relating the fields of the given transducer to those of an adjoint transducer. The material parameters of the adjoint transducer are determined by certain transpositions and interchanges of the material parameters of the given transducer. The reciprocity lemma leads to the generalized reciprocal relations between the characteristic (PWSM and SIM) matrices of the given and adjoint transducers. In addition, corollaries of the adjoint reciprocity relations analogous to conventional reciprocity theorems are derived for adjoint pairs of transducers.

Power relations and the associated "value classes" are investigated also for electroacoustic transducers and their adjoints. In particular, it is shown that the dissipative characteristics (lossiness, losslessness, or "gaininess") of the given transducer are preserved point wise in the adjoint transducers.

Although a unique adjoint corresponds to a given operator, many different operators can represent the equations of the same transducer. For example, multiplication of one of the equations of motion by a constant will change the operator matrix without changing the content of the equation of motion. Analysis shows, however, that for a given transducer only two operators, $L^{+}$and $L^{-}$(from here on the " + " will be omitted from $L^{+}$), have an associated adjoint operator, $L^{\prime \prime}$ and $L^{--"}$, which represents the equations of another transducer (the adjoint transducer). A transducer selfadjoint $\left(L^{\prime \prime}=L\right.$ or $L^{-1}=L^{-}$) with respect to $L$ or $L^{-}$corresponds to the conventional reciprocal or antireciprocal transducer respectively. One of the advantages of the linear operator approach is that it facilitates the identification of self-adjoint transducers.

A number of experimental measurement techniques applicable to reciprocal (or antireciprocal) transducers only, could be extended to nonreciprocal transducers if the adjoint transducers were producible in the laboratory. In general, the difficulties in synthesizing the necessary electroacoustic material parameters may discourage or prevent the creation of the adjoint transducers. However, two groups of transducers are discussed for which the creation of an adjoint transducer involves, in principle, a simple modification of the given transducer.

\section{The Transducer System}

The electroacoustic transducer system under consideration is pictured in figure 1. The transducer is bounded by the closed, finite surface $A_{0}$. The em power supply or detector, which is bounded by the closed, finite surface $A_{\mu}$, feeds the transducer through the area $S_{0}$ common to both $A_{0}$ and $A_{\mu}$. The transducers, which may contain static bias fields and their sources, are termed "passive" if they cannot radiate more power than they absorb. The present analysis, however, is not limited to passive transducers, but may include transducers which are active because they contain "gainy" material, or radiating sources, or both. (A transducer will be called source-free if its internal equations of motion are homogeneous, i.e., if they are satisfied by all fields equal to zero.)

The transducer-plus-(power supply or detector) is immersed in a homogeneous, isotropic, stationary fluid which extends to infinity and supports the time harmonic $\left(e^{-i \omega t}, \omega>0\right)$ acoustic pressure-velocity field of small (first order differential) amplitude. The static pressure, static density, and propagation constant (the latter two of which may be complex function of real frequency $\omega$ 
to account for viscous and "expansive friction" losses) are constants in space and time denoted by $P_{0}, \rho_{0}$ and $k$ respectively. The fluid between the infinite $x y$ plane $S_{1}(z=0)$ and the external surface

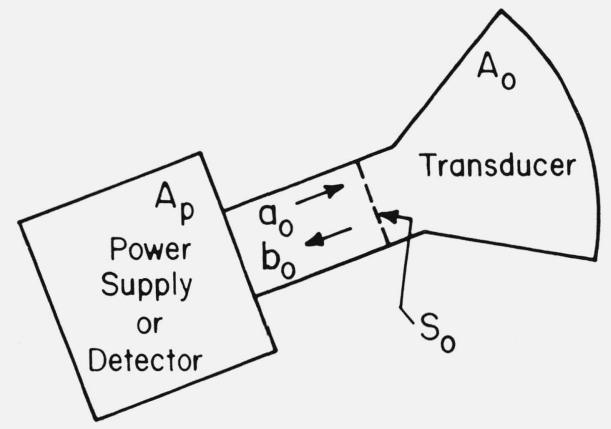

$P_{0}, \rho_{0}, k$

FIGURE 1. Schematic of the transducer system.

$A=A_{o}+A_{p}$ denotes the external surface of the electroacoustic transducer-plus-(power supply or detector).

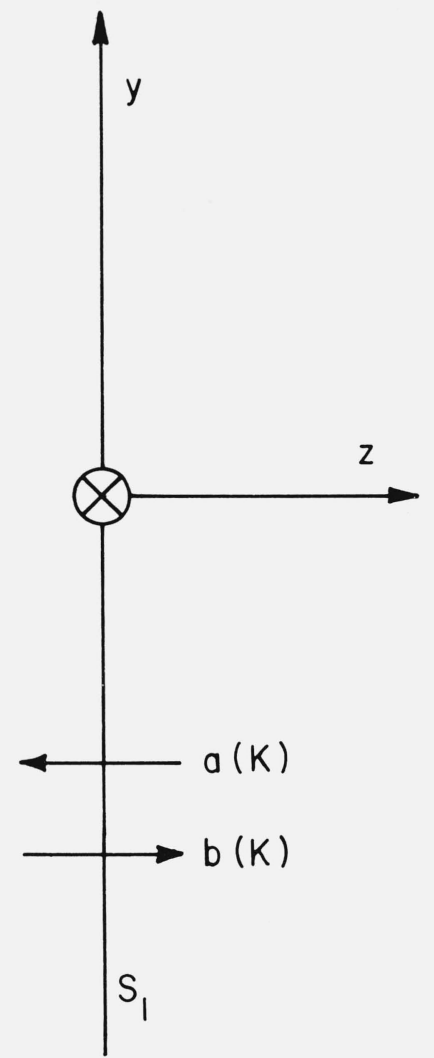

$A$ of the transducer-plus-(power supply or detector) contains no acoustic sources, ${ }^{2}$ although arbitrary sources (at frequency $\omega$ ) may exist to the right of $S_{1}$.

The normal velocity on the surface $A_{p}$ of the power supply-detector, including the feed area $\mathrm{S}_{0}$, is assumed negligible. Electromagnetic shielding necessary for deriving the adjoint reciprocity relations is discussed in section 5 .

The area $S_{0}$ may designate the perpendicular cross section of an open or closed em waveguide (uniform and isotropic) in which a single em mode is propagating with incident amplitude $a_{0}$ and emergent amplitude $b_{0}$, or simply the cross section of two wire leads when the frequency $\omega$ is low enough to allow analysis by quasistatic voltages and currents. The cross-sectional area $S_{0}$ for a mode on an open waveguide (such as a Lecher line or microstrip) must extend sufficiently far from the guide to insure that the fields in the mode outside $S_{0}$ contribute negligibly to the normalization integral ((7) below). The surfaces $A_{p}, S_{0}$, and $A_{0}$ represent imaginary boundaries in that they are chosen to facilitate the theoretical analysis and need not coincide with the physical boundaries of the system. Of course, the surface $A=A_{0}+A_{p}$ must be chosen within (or on the interior boundary of) the ambient fluid.

The continuity and momentum equations, which govern the harmonic acoustic fields in the source-free fluid between the surface $A$ and the infinite plane $S_{1}$, may be expressed as

$$
\begin{aligned}
& k^{2} p=-i \omega \rho_{0} \nabla \cdot \bar{u} \\
& \nabla p=i \omega \rho_{0} \bar{u},
\end{aligned}
$$

${ }^{2}$ Between $A$ and $S$, there can be finite regions of either acoustically rigid material or material in which volume forces are zero and stress is linearly related to strain by a symmetric Hooke's tensor. However, the reference plane $S_{1}$ must remain entirely in the homogeneous ambient fluid. 
where the complex amplitudes, $p$ and $\bar{u}$, denote the position-dependent excess pressure and fluid velocity. The effect of gravity on harmonic variation in mass density is neglected.

The excess pressure and normal velocity on the surface $S_{1}$ may be expanded in a double Fourier integral (transform) over plane wave voltage and current spectral densities [4]:

$$
\begin{gathered}
p(\bar{R})=\frac{1}{2 \pi} \int_{\bar{K}} V(\bar{K}) e^{i \bar{K} \cdot \bar{R}} d \bar{K} \\
u_{z}(\bar{R})=-\frac{1}{2 \pi} \int_{\bar{K}} I(\bar{K}) e^{i \bar{K} \cdot \bar{R}} d \bar{K} .
\end{gathered}
$$

Conversely, the plane-wave voltage and current densities, which are defined in terms of the incoming $a(\bar{K})$ and outgoing $b(\bar{K})$ acoustic plane-wave amplitudes,

$$
\begin{aligned}
V(\bar{K}) & =a(\bar{K})+b(\bar{K}) \\
I(\bar{K}) & =[a(\bar{K})-b(\bar{K})] \eta(K),
\end{aligned}
$$

may be expanded in a double Fourier integral (inverse transform) over the excess pressure and normal velocity:

$$
\begin{aligned}
& V(\bar{K})=\frac{1}{2 \pi} \int_{S_{1}} p(\bar{R}) e^{-i \bar{K} \cdot \bar{R}} d \bar{R} \\
& I(\bar{K})=-\frac{1}{2 \pi} \int_{S_{1}} u_{z}(\bar{R}) e^{-i \bar{K} \cdot \bar{R}} d \bar{R} .
\end{aligned}
$$

The vector $\bar{K}=K_{x} \hat{\imath}+K_{y} \hat{j}$ can be interpreted as the transverse part of the plane-wave propagation vector $\bar{k}$. The vector $R$ is confined to the $x y$ plane $S_{1}$, and $\eta(K)$ equals $\gamma / \omega \rho_{0}$, where $\gamma$ is defined as $\left(k^{2}-K^{2}\right)^{1 / 2}$ with the sign of the radical chosen to keep the real and imaginary parts of $\gamma$ positive.

The transverse em fields $\left(\bar{E}_{t}, \bar{H}_{t}\right)$ on the cross section $S_{0}$ of a waveguide may be written as the sum of the transverse electric $\bar{e}_{0}$ and magnetic $\bar{h}_{0}$ components of the mode traveling to the right with incident amplitude $a_{0}$ and to the left with emergent amplitude $b_{0}$ :

where

$$
\bar{E}_{t}=V_{0} \bar{e}_{0}, \quad \bar{H}_{t}=I_{0} \bar{h}_{0},
$$

$$
\begin{aligned}
& V_{0}=a_{0}+b_{0} \\
& I_{0}=\left(a_{0}-b_{0}\right) \eta_{0} .
\end{aligned}
$$

If the dimensions of $\bar{e}_{0}$ and $\bar{h}_{0}$ are chosen as (meter) $)^{-1}$ and $\left(\bar{e}_{0}, \eta_{0} \bar{h}_{0}\right)$ are consistent with Maxwell's equations, $V_{0}$ behaves dimensionally as volts, $I_{0}$ as amperes, and $\eta_{0}$ as an admittance $\left(\mathrm{ohm}^{-1}\right)$ normally chosen as a positive real number. Moreover, normalization may be expressed as a nondimensional number equal to unity, i.e.,

$$
\int_{S_{0}}\left(\bar{e}_{0} \times \bar{h}_{0}\right) \cdot \hat{n} \mathrm{da}=1,
$$

where $\hat{n}$ is the inward normal to the transducer. (The rationalized MKS system of units is used throughout.) If the area $S_{0}$ simply cuts two wire leads at quasi-static frequencies, $V_{0}$ and $I_{0}$ refer 
to conventional circuit voltages and currents which do not necessarily serve as genuine modal coefficients. In that case, (6) become a definition of $a_{0}$ and $b_{0}$.

The PWSM description relates the outgoing amplitudes, $b_{0}$ and $b(\bar{K})$, to the incoming amplitudes, $a_{0}$ and $a(\bar{K})$, by the scattering equations (see ref. [4]),

$$
\begin{gathered}
b_{0}=S_{00} a_{0}+\int_{L^{\circ}} S_{01}(\bar{L}) a(\bar{L}) d \bar{L} \\
b(\bar{K})=S_{10}(\bar{K}) a_{0}+\int_{L} S_{11}(\bar{K}, \bar{L}) a(\bar{L}) d \bar{L}
\end{gathered}
$$

The scattering matrix is resolved into the four submatrices $S_{01}, S_{10}, S_{11}$ and $S_{00}$ which represent the receiving, radiating, acoustic scattering, and em reflection properties respectively for the electroacoustic transducer. Like $\bar{K}$, the vector $\bar{L}$ can be interpreted as the transverse part of the propagation vector $\bar{l}$.

Alternatively, the SIM relations ${ }^{3}$ characterize the external behavior of the transducer by relating the "voltage" $V_{0}$ and excess pressure $p$ on the surface of the transducer $A_{0}$ (except possibly on the feed area $S_{0}$ ) to the "current" $I_{0}$ and the inward (to transducer) normal velocity $u_{n}$ on the surface $A_{0}$ :

$$
\begin{gathered}
V_{0}=Z_{b} I_{0}+\int_{A_{0}} h^{\prime}\left(\bar{r}_{0}\right) u_{n}\left(\bar{r}_{0}\right) d a_{0} \\
p(\bar{r})=h(\bar{r}) I_{0}+\int_{A_{0}} Z_{0}\left(\bar{r}, \bar{r}_{0}\right) u_{n}\left(\bar{r}_{0}\right) d a_{0} .
\end{gathered}
$$

Although three of the four spatial impedances $\left(Z_{b}, h^{\prime}, h, Z_{0}\right)$ depend upon the position vectors $\left(\bar{r}, \bar{r}_{0}\right)$ to the surface $A_{0}$ and all four depend upon the harmonic time frequency, they are assumed independent of the medium or sources surrounding the transducer and, as linearity implies, independent of $V_{0}, I_{0}, p$ and $u_{n}$.

It can be shown [9], without reference to the internal behavior of the transducers, that the PWSM equations (8) imply the SIM relations (9) but not vice versa. That is, the PWSM description applies to a larger class of transducers than do the SIM relations. However, the alternate expressions of reciprocity (and antireciprocity),

$$
\begin{gathered}
\eta_{0} S_{01}(\bar{K})= \pm \eta(K) S_{10}(-\bar{K}) \\
\eta(K) S_{11}(\bar{K}, \bar{L})=\eta(L) S_{11}(-\bar{L}, \bar{K}),
\end{gathered}
$$

and

$$
\begin{gathered}
h^{\prime}(\bar{r})= \pm h(\bar{r}) \\
Z_{0}\left(\bar{r}, \bar{r}_{0}\right)=Z_{0}\left(\bar{r}_{0}, \bar{r}\right)[6,7],
\end{gathered}
$$

which are derived in section 5, can be proven [9] equivalent for transducers which obey both descriptions.

In analogy to the 2-port cases discussed by McMillan [10], Kerns [4] defines reciprocity, antireciprocity, and nonreciprocity for electroacoustic transducers in terms of whether (10a) holds with the plus sign, the minus sign, or not at all. Foldy and Primakoff $[6,7]$ do not use the term "antireciprocity" explicitly, but distinguish between the plus and minus sign in (1la) by con-

${ }^{3}$ Foldy and Primakoff refer to equations (9) as the "linearity relations." Here they are referred to as the "spacial impedance-matrix relations" to avoid confusion with the spectral scattering-matrix equations (8), which are also linear. 
sidering reciprocity through electric-type coupling and magnetic-type coupling.

Equations which characterize the internal behavior of the electroacoustic transducers under consideration are introduced in the following section.

\section{The Operator Matrix Description}

Primakoff and Foldy [7], in their original work on electroacoustic reciprocity, examined the equations which model the internal operation of a large class of linear transducers. They found that if certain "symmetry conditions" were satisfied by its material parameters and static fields, the transducers obeyed the SIM reciprocity relations (11). The present derivation begins in a similar way by writing the linear, harmonic $\left(e^{-i \omega t}\right)$ equations that govern the "physics" of the material within the transducer $A_{0}$ :

$$
\begin{gathered}
\nabla \cdot \overline{\bar{T}}+i \omega \rho_{m}^{\circ} \bar{u}+\bar{f}=0 \\
\nabla \times \bar{E}-i \omega \bar{B}=0 \\
\nabla \times \bar{H}+i \omega \bar{D}-\bar{J}=0 .
\end{gathered}
$$

The momentum equation (12) expresses Newton's second law for stationary $\left(\bar{u}_{0}=0\right)$ media, and (13) express Maxwell's equations in differential form for stationary media in which regions of electric and magnetic polarization may exist.

The static quantities, which will be distinguished by the superscript or subscript " 0 ," are real functions of the space coordinates. All other quantities, except the real frequency $\omega$, represent complex, space-dependent, harmonic amplitudes, which superimpose upon the static variables as products with $e^{-i \omega t}$. For example, if the total stress dyadic at a point in space and time is denoted by $\overline{\bar{T}}^{\prime}(\bar{r}, t)$ then $\overline{\bar{T}}^{\prime}$ can be divided into a real static part $\overline{\bar{T}}_{0}(\bar{r})$ and a complex harmonic part $\overline{\bar{T}}(\bar{r}) e^{-i \omega t}$ i.e.,

$$
\overline{\bar{T}}^{\prime}(\bar{r}, t)=\overline{\bar{T}}_{0}(\bar{r})+\overline{\bar{T}}(\bar{r}) e^{-i \omega t} .
$$

Of course, it is the real part of $\overline{\bar{T}}^{\prime}$ that corresponds to a measurable quantity. Only the complex harmonic amplitude $\overline{\bar{T}}(\bar{r})$ shows in (12). Similarly the total mass density $\rho_{m}^{\prime}(\bar{r}, t)$ may be divided as

$$
\rho_{m}^{\prime}(\bar{r}, t)=\rho_{m}^{0}(\bar{r})+\rho_{m}(\bar{r}) e^{-i \omega t},
$$

but only the real static part occurs in (12). In fact, the mass continuity equation is not included in addition to the momentum equation (12) because it merely introduces the extra variable $\rho_{m}(\bar{r})$.

The other variables in (12) are $\bar{u}$, the harmonic velocity of the material, and $\bar{f}$, the total harmonic volume force. The usual harmonic em field vectors are denoted by $\bar{E}, \bar{H}, \bar{B}, \bar{D}$, and $\bar{J}$. All variables refer to macroscopic quantities which are evaluated, at least in concept, by averaging microscopic variables over small but finite volumes and time intervals.

The number of unknowns in (12) and (13) reduces to the number of equations if the following linear constitutive relations characterize the transducer material:

$$
\begin{aligned}
& \bar{D}=\overline{\bar{\epsilon}} \cdot \bar{E}+\overline{\bar{\tau}} \cdot \bar{H}+\overline{\bar{\alpha}}: \overline{\bar{s}}+\bar{P}_{s} \\
& \bar{B}=\overline{\bar{\nu}} \cdot \bar{E}+\overline{\bar{\mu}} \cdot \bar{H}+\overline{\bar{\beta}}: \overline{\bar{s}}+\mu_{0} \bar{M}_{s} \\
& \bar{J}=\overline{\bar{\sigma}} \cdot\left(\bar{E}+\bar{u} \times \bar{B}_{0}\right)+\rho_{e}^{0} \bar{u}^{\prime}+\bar{J}_{s} \\
& \overline{\bar{T}}=\overline{\overline{\bar{c}}}: \overline{\bar{s}}-\overline{\bar{\alpha}} \cdot \bar{E}-\overline{\overline{\bar{\beta}}} \cdot \bar{H}
\end{aligned}
$$




$$
\bar{f}=\rho_{e}^{0} \bar{E}+\bar{J} \times \bar{B}_{0}+\bar{f}_{s},
$$

where the strain dyadic $\overline{\bar{s}}$ is related to the velocity by

$$
\overline{\bar{s}}=-\frac{1}{i \omega}\left(\nabla \bar{u}-\frac{1}{2}(\nabla \times \bar{u}) \times \overline{\bar{I}}\right) .
$$

$(\overline{\bar{I}}$ is the identity dyadic).

The various "dot products" involved in the vector and dyadic transformations of (14-19) can be understood in terms of Cartesian tensor notation, in which $\overline{\bar{\epsilon}} \cdot \bar{E}, \overline{\bar{\alpha}} \cdot \bar{E}, \overline{\bar{\alpha}}: \overline{\bar{s}}$ and $\overline{\overline{\bar{c}}}: \overline{\bar{s}}$ become $\epsilon_{i j} E_{j}, \alpha_{i j k}^{\prime} E_{k} \cdot \alpha_{i j k} s_{j k}$, and $c_{i j k l} s_{k l}$ respectively (summation over repeated dummy indices is implied). The " $n$-adic" notation [11] is used wherever convenient because it preserves the familiar vector-dyadic notation for the electroacoustic fields [7, 12].

The dyadics $\overline{\bar{\sigma}}, \overline{\bar{\epsilon}}, \overline{\bar{\mu}}$, and $(\overline{\bar{\tau}}, \overline{\bar{v}})$ stand for the conductivity, permittivity, permeability, and magneto-electric properties [13] of the transducer material.

The direct piezoelectric and piezomagnetic stress triadics [14], $\overline{\bar{\alpha}}$ and $\overline{\bar{\beta}}$, may be chosen symmetric:

$$
\begin{aligned}
& \alpha_{i j k}=\alpha_{i k j} \\
& \beta_{i j k}=\beta_{i k j},
\end{aligned}
$$

since the strain dyadic is symmetric,

$$
s_{j k}=\frac{-1}{2 i \omega}\left\{\frac{\partial u_{j}}{\partial x_{k}}+\frac{\partial u_{k}}{\partial x_{j}}\right\}=s_{k j} .
$$

Similarly, because the stress is a symmetric dyadic $\left(T_{i j}=T_{j i}\right)$ (provided there are no distributions of body torque, stress couples, or angular momentum density [15]), the converse piezoelectric and piezomagnetic stress triadics [14], $\overline{\bar{\alpha}}^{\prime}$ and $\overline{\bar{\beta}}^{\prime}$, must possess the symmetry,

$$
\begin{aligned}
& \alpha_{i j k}^{\prime}=\alpha_{j i k}^{\prime} \\
& \beta_{i j k}^{\prime}=\beta_{j i k}^{\prime} .
\end{aligned}
$$

The symmetry of the stress dyadic also requires the Hooke's tetradic $\overline{\bar{c}}$ to obey the symmetry

$$
c_{i j k l}=c_{j i k l}
$$

and the symmetry of the strain dyadic allows the defined symmetry

$$
c_{i j k l}=c_{i j l k} .
$$

The static charge distribution $\rho_{e}^{0}$, the static magnetic induction $\bar{B}_{0}$, all other static quantities, and material parameters which may depend upon frequency $\omega$, remain independent of the values of the harmonic variables.

Although only transducers which obey the homogeneous equations are considered finally (source-free transducers), the harmonic sources of polarization $\bar{P}_{s}$, magnetization $\bar{M}_{s}\left(\mu_{0}\right.$ is the permeability of free-space), current $\bar{J}_{s}$, and volume force $\bar{f}_{s}$ have been included in (14), (15), (16), and (18) to allow insight into applications involving these sources.

A number of other assumptions underlie the development of (14-19). In the current equation (16) the "Hall effect" of the magnetic induction on drift velocity is ignored, as well as harmonic variations in conductivity which might be found, for example, in a biased carbon microphone. 
The volume forces on electric and magnetic dipoles, static currents, and harmonic variations in mass density (acted upon by gravity) have been omitted in the force equation (18). The effects of gravity are usually minute, and if the material that contains the dipoles or static currents is rigid or has insignificant effect on the essential behavior of the transducer, the volume forces on the dipoles and static currents are justifiably neglected also. The magnet in a dynamic speaker may experience appreciable volume forces, but because the magnet is relatively rigid, (12-19) describe such speakers. However, the operation of a hypothetical transducer containing dipole or static current elements which move through strongly inhomogeneous em fields would not, in general, be described by (18).

Application of Maxwell's stress tensor reveals that forces on surface charges and currents can be evaluated by substituting the appropriate surface delta functions into (18) if nonconductors contain no static surface charge, and if the electric field just outside the surface of conductors, on which static surface charge exists, is used in the evaluation of $\rho_{e}^{0} \bar{E}$. The force term $\rho_{e} \bar{E}_{0}$ is not needed in (18) because it vanishes in nonconductors where the harmonic charge distribution $\rho_{e}$ must be zero, and in conductors where the static electric field $\bar{E}_{0}$ must be zero. The standard condenser microphone is well described by (12-19).

The constitutive equations (14-19) may be used to reduce the three physical laws (12), (13a), and (13b) to functions of three vectors, the electric field $\bar{E}$, the magnetic field $\bar{H}$, and the velocity field $\bar{u}$. The final three equations are conveniently displayed in operator matrix form as

$$
\left.\begin{array}{lll}
(i \omega \overline{\bar{\epsilon}} \cdot-\overline{\bar{\sigma}} \cdot) & (\nabla \times+i \omega \overline{\bar{\tau}} \cdot) & \left(-\overline{\bar{\alpha}}: \nabla+\left(\overline{\bar{\sigma}} \times \bar{B}_{0}\right) \cdot-\rho_{e}^{0}\right) \\
(\nabla \cdot \times-i \omega \overline{\bar{\nu}} \cdot) & (-i \omega \overline{\bar{\mu}} \cdot) & (\overline{\bar{\beta}}: \nabla) \\
\left(\nabla \cdot \overline{\bar{\alpha}}^{\prime} \cdot+\bar{B}_{0} \times \overline{\bar{\sigma}} \cdot-\rho_{e}^{0}\right) & \left(\nabla \cdot \overline{\bar{\beta}}^{\prime} \cdot\right) & \left(\frac{\nabla \cdot \overline{\bar{c}}: \nabla}{i \omega}-\left(\bar{B}_{0} \times \overline{\bar{\sigma}}^{\prime} \times \bar{B}_{0}\right) \cdot+\rho_{e}^{0} \bar{B}_{0} \times-i \omega \rho_{m}^{0}\right)
\end{array}\right]\left[\begin{array}{l}
\bar{E} \\
\bar{H}
\end{array}\right]=
$$

Since $(\nabla \times \bar{u}) \times \overline{\bar{I}}$ is antisymmetric, its dot products with the symmetric $\overline{\bar{\alpha}}, \overline{\bar{\beta}}$, and $\overline{\overline{\bar{c}}}$ vanish. All operator symbols of the $3 \times 3$ matrix containing three vectors are evaluated in succession from right to left. In full scalar matrix notation, the left side of (24) becomes a $9 \times 9$ matrix operating on a 9 . element column matrix, and the right side another 9-element column matrix.

The particular arrangement of elements in the matrix of (24) was chosen because it exhibits Maxwell's equations as a $2 \times 2$ submatrix (the upper left corner), and because the associated adjoint operator matrix also represents the equations of an electroacoustic transducer (the adjoint transducer).

\section{The Adjoint Operator Matrix and an Electroacoustic Reciprocity Lemma}

The development of the desired generalized reciprocity relations requires the intermediate derivation of a bilinear divergence expression which combines, essentially, the Lorentz lemma [16] 
of electromagnetic theory with the acoustical reciprocity theorem ((45) below). The theory of adjoint operators assures the existence of such a bilinear expression (called the "bilinear identity"), and provides a straightforward method for obtaining the identity from the operator matrix of (24) [17]. Specifically, if $L$ denotes the linear operator matrix of (24), the bilinear identity may be written in differential form as

$$
\phi_{1 t} L \phi_{2}-\phi_{2 t} L^{\prime \prime} \phi_{1}=\nabla \cdot \bar{F}
$$

where $L^{\prime \prime}$ denotes the adjoint operator associated with $L ; \phi_{1}$ and $\phi_{2}$ are any two continuously differentiable column matrices containing three vectors (the subscript $t$ transposes the column matrix to the corresponding row matrix); and $\nabla \cdot \bar{F}$ is the required divergence expression. The explicit evaluation of the bilinear concomitant $\bar{F}$ requires the determination of the adjoint operator $L^{\prime \prime}$, which is actually defined by (25) [17].

For a given linear operator $L$, the adjoint operator $L^{\prime \prime}$ exists uniquely [9], and may be found by transposing the matrix elements of $L$, and replacing the differential operators by their adjoints. ${ }^{4}$ Fortunately, the adjoints of the four different linear differential operators of $L$,

$$
\nabla \times, \overline{\bar{\alpha}}: \nabla, \nabla \cdot \overline{\overline{\bar{\alpha}^{\prime}}} \cdot \text {, and } \nabla \cdot \overline{\overline{\bar{c}}}: \nabla
$$

are readily determined as

$$
\nabla \times,-\nabla \cdot \overline{\bar{\alpha}}_{t} \cdot,-\overline{\bar{\alpha}}_{t}^{\prime}: \nabla \text {, and } \nabla \cdot \overline{\bar{c}}_{t}: \nabla
$$

respectively, with the help of the associated identities,

$$
\begin{gathered}
\bar{A}_{1} \cdot\left(\nabla \times \bar{A}_{2}\right)-\bar{A}_{2} \cdot\left(\nabla \times \bar{A}_{1}\right)=\nabla \cdot\left(\bar{A}_{2} \times \bar{A}_{1}\right) \\
\bar{A}_{1} \cdot\left(\nabla \cdot\left(\overline{\bar{\alpha}} \cdot \bar{A}_{2}\right)\right)+\bar{A}_{2} \cdot\left(\overline{\bar{\alpha}}_{t}: \nabla \bar{A}_{1}\right)=\nabla \cdot\left(\overline{A_{1}} \cdot \overline{\bar{\alpha}} \cdot \bar{A}_{2}\right) \\
\bar{A}_{1} \cdot\left(\nabla \cdot\left(\overline{\overline{\bar{c}}}: \nabla \bar{A}_{2}\right)\right)-\bar{A}_{2} \cdot\left(\nabla \cdot\left(\overline{\overline{\bar{c}}_{t}} \pm \nabla \bar{A}_{1}\right)\right)=\nabla \cdot\left(\bar{A}_{1} \cdot \overline{\bar{c}}: \nabla \bar{A}_{2}-\bar{A}_{2} \cdot \overline{\overline{\bar{c}}}_{t}: \nabla \bar{A}_{1}\right) .
\end{gathered}
$$

The subscript $t$ denotes the transposed matrix, and $\bar{A}_{1}, \bar{A}_{2}$ afe arbitrary, continuously differentiable vectors. In tensor notation the transpose of $\alpha_{i j k}$ and $c_{i j k l}$, for example, used in (27), (28) and below is $\alpha_{j k i}$ and $c_{k l i j}$. Note that the curl operator is self-adjoint, that the direct and converse piezoelectric and piezomagnetic operators are adjoints of each other if $\overline{\bar{\alpha}}_{t}=-\overline{\bar{\alpha}}^{\prime}, \overline{\bar{\beta}}=-\overline{\bar{\beta}_{t}^{\prime}}$, and that the purely acoustic operator is self-adjoint if $\overline{\overline{\bar{c}}}_{t}=\overline{\overline{\bar{c}}}$. These self-adjoint properties are understandable from Lanczos' theory of adjoint operators where he shows that the curl operator is self-adjoint and that the gradient and divergence operators are negative adjoints of each other [17].

The straightforward transposition of the nondifferential elements of $\mathrm{L}$ completes the construction of the adjoint operator $L^{\prime \prime}$ :

$$
\begin{aligned}
& \left.\left(i \omega \overline{\bar{\epsilon}}_{t} \cdot-\overline{\bar{\sigma}}_{t} \cdot\right) \quad\left(\nabla \times-i \omega \overline{\bar{\nu}}_{t} \cdot\right) \quad-\overline{\bar{\alpha}}_{t}^{\prime}: \nabla-\overline{\bar{\sigma}}_{t} \times \bar{B}_{0}\right) \cdot-\rho_{e}^{0} \\
& L^{a}=\left[\begin{array}{lll}
\left(\nabla \times+i \omega \overline{\bar{\tau}}_{t}:\right) & \left(-i \omega \overline{\bar{\mu}}_{t}:\right) \quad\left(-\overline{\bar{\beta}}_{t}^{\prime}: \nabla\right)
\end{array}\right.
\end{aligned}
$$

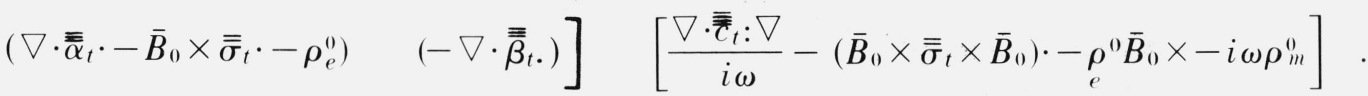

Comparison of the adjoint operator $L^{a}$ with $L$ reveals that, at a given frequency $\omega$, the adjoint

4 Determination of adjoint differential operators as implied by the bilinear identity (25) is not synonymous with the interchanging of rows and columns of the Cartesian matrix form of the operator. Contrast, for example, Lanczos, ref. [17] with Auld, ref. [12] 
operator describes the equations of a second transducer (the adjoint transducer) in which the transposed parameters

$$
\overline{\bar{\sigma}}_{t}, \overline{\bar{\epsilon}}_{t}, \overline{\bar{\mu}}_{t},-\overline{\bar{\tau}}_{t},-\overline{\bar{\nu}}_{t}, \overline{\bar{\alpha}}_{t},-\beta_{t}, \overline{\bar{\alpha}}_{t}^{\prime},-\overline{\bar{\beta}}_{t}^{\prime}, \overline{\overline{\bar{c}}}_{t},-\bar{B}_{0}, \rho_{e}^{0}, \text { and } \rho_{m}^{0}
$$

replace the original parameters

$$
\overline{\bar{\sigma}}, \overline{\bar{\epsilon}}, \overline{\bar{\mu}}, \overline{\bar{\nu}}, \overline{\bar{\tau}}, \overline{\bar{\alpha}}^{\prime}, \overline{\bar{\beta}}^{\prime}, \overline{\bar{\alpha}}, \overline{\bar{\beta}}, \overline{\overline{\bar{c}}}, \bar{B}_{0}, \rho_{e}^{0} \text { and } \rho_{m}^{0},
$$

respectively. The adjoint transducer refers to a mathematically conceived transducer with hypothetical material parameters which may or may not be physically realizable (see sec. 7 ). When the transposed parameters (30a) equal the original parameters $(30 \mathrm{~b})$, the operators $L$ and $L^{\prime \prime}$ become identical to form a self-adjoint operator for which the adjoint transducer and original transducer are one and the same. In that case $\bar{B}_{0}$ is zero $\left(\bar{B}_{0}=-\bar{B}_{0}\right)$, which corresponds to the electrictype coupling of Primakoff and Foldy [7].

The expression (29) for the adjoint operator allows the explicit evaluation of $\nabla \cdot \bar{F}$. If $\phi_{2}$ is restricted to solutions of (24), and $\phi_{1}$ to solutions of the adjoint equations corresponding to (24) with $L^{\text {" }}$ replacing $L$, i.e.,

$$
L \phi_{2}=Q, L^{a} \phi_{1}=Q^{a}
$$

where

$$
\phi_{2}=\left[\begin{array}{c}
\bar{E} \\
\bar{H} \\
\bar{u}
\end{array}\right], \quad \phi_{1}=\left[\begin{array}{c}
\bar{E}^{\prime \prime} \\
\bar{H}^{\prime \prime} \\
\bar{u}^{\prime \prime}
\end{array}\right]
$$

and

$$
Q=\left[\begin{array}{l}
\bar{J}_{s}-i \omega \bar{P}_{s} \\
i \omega \mu_{0} \bar{M}_{s} \\
\bar{f}_{s}-\bar{B}_{0} \times \bar{J}_{s}
\end{array}\right], Q^{\prime \prime}=\left[\begin{array}{l}
\bar{J}_{s}^{\prime \prime}-i \omega \bar{P}_{s}^{\prime \prime} \\
i \omega \mu_{0} \bar{M}_{s}^{\prime \prime} \\
\bar{f}_{s}^{\prime \prime}+\bar{B}_{0} \times \bar{J}_{s}^{\prime \prime}
\end{array}\right]
$$

then $\nabla \cdot \bar{F}$ becomes, after rearrangement and use of (17) and (19),

$$
\begin{aligned}
\nabla \cdot \bar{F} & =\nabla \cdot\left(\bar{E} \times \bar{H}^{\prime \prime}-\bar{E}^{\prime \prime} \times \bar{H}\right)-\nabla \cdot\left(\bar{u}^{\prime \prime} \cdot \overline{\bar{T}}-\bar{u} \cdot \overline{\bar{T}}^{\prime \prime}\right) \\
& =\bar{E}^{\prime \prime} \cdot\left(\bar{J}_{s}-i \omega \bar{P}_{s}\right)+i \omega \mu_{0} \bar{H}^{\prime \prime} \cdot \bar{M}_{s}+\bar{u}^{\prime \prime} \cdot\left(\bar{f}_{s}-\bar{B}_{0} \times \bar{J}_{s}\right) \\
& -\bar{E} \cdot\left(\bar{J}_{s}^{\prime \prime}-i \omega \bar{P}_{s}^{\prime \prime}\right)-i \omega \mu_{0} \bar{H} \cdot \bar{M}_{s}^{\prime \prime}-\bar{u} \cdot\left(\bar{f}_{s}^{\prime \prime}+\bar{B}_{0} \times \bar{J}_{s}^{\prime \prime}\right) .
\end{aligned}
$$

When the harmonic sources are set equal to zero (in the transducer not the power supply), application of the divergence theorem to (34) in the volume bounded by the surface $A_{0}$ of the transducer yields a generalized reciprocity "lemma" between the independent electroacoustic fields of a given transducer and those of its mathematical adjoint:

$$
\int_{A_{0}}\left(\bar{E} \times \bar{H}^{\prime \prime}-\bar{E}^{\prime \prime} \times \bar{H}\right) \cdot \hat{n} d a+\int_{A_{0}}\left(\overline{\bar{T}}^{\prime \prime} \cdot \bar{u}-\overline{\bar{T}} \cdot \bar{u}^{\prime \prime}\right) \cdot \hat{n} d a=0 .
$$

The name "generalized electroacoustic reciprocity lemma" seems appropriate for (35) since it represents a merger of the Lorentz lemma [16] of em theory and the acoustical reciprocity theorem ( (45) below) as applied to adjoint systems. For self-adjoint transducers, i.e , transducers for which the transposed material parameters of (30a) equal those of $(30 \mathrm{~b})$, (35) reduces to the 
corresponding Primakoff-Foldy expression [7] in which a second set of fields on the same transducer replace the adjoint fields. Auld [12] uses this latter expression with the complex conjugate fields to derive the orthogonality relation [18] between different modes of piezoelectric waveguides and resonators. Equation (35) demonstrates that the waveguides and resonators need not be restricted to piezoelectric material in order for the modes to satisfy orthogonality.

A more general expression similar to (35) may be derived from the equations represented by the operator $L$. Each row of the operator $L$ in (24) could be multiplied by an arbitrary constant without affecting the essential content of the equations. Specifically, if the first, second, and third row of $L$ are multiplied by arbitrary complex constants, $c_{1}, c_{2}$, and $c_{3}$ respectively, (35) is replaced by

$$
\int_{A_{0}}\left[c_{1}\left(\bar{E} \times \bar{H}^{a}\right)-c_{2}\left(\bar{E}^{a} \times \bar{H}\right)\right] \cdot \hat{n} d a+c_{3} \int_{A_{0}}\left(\overline{\bar{T}}^{a} \cdot \bar{u}-\overline{\bar{T}} \cdot \bar{u}^{a}\right) \cdot \hat{n} d a=0 .
$$

Although (36) has mathematical validity and interest, the constants $c_{1}, c_{2}$, and $c_{3}$ must be restricted if (36) is to be used in the derivation of the generalized reciprocity relations between a transducer and its adjoint transducer. (The adjoint fields will depend upon $c_{1}, c_{2}, c_{3}$.) First, because the right side of (36) is zero, the constant $c_{1}$, can be set equal to unity without loss of generality. Second, $c_{2}$ must equal $c_{1}=1$ to accomplish the derivation of the scattering-matrix reciprocity relations ((53) below). And third, if the adjoint operator underlying (36) is required to describe the fields of a second transducer (the adjoint transducer), comparison of (29) with (24) (when the constants $c_{1}, c_{2}$, and $c_{3}$ are included) shows that for $c_{1}=c_{2}=1$, the only allowable values of $c_{3}$ are \pm 1 unless both $\bar{B}_{0}$ and $\rho^{0}{ }_{e}$ are zero. If both $\bar{B}_{0}$ and $\rho^{0}{ }_{e}$ vanish throughout the transducers, the adjoint operator represents an adjoint transducer for all values of $c_{3}$.

Equation (35) already represents the case of $c_{3}=+1$, where the relationship between the adjoint transducer parameters and those of the original transducer is given by (30).

For $c_{3}=-1$, the operator $L$ in (24) changes to $L^{-}$, the superscript $(-)$indicating that the third row of $L$ has been multiplied by a minus sign. The operator adjoint to $L^{-}$describes an adjoint transducer in which the transposed parameters

$$
\overline{\bar{\sigma}}_{t}, \overline{\bar{\epsilon}}_{t}, \overline{\bar{\mu}}_{t},-\overline{\bar{\tau}}_{t},-\overline{\bar{v}}_{t},-\overline{\bar{\alpha}}_{t}, \overline{\bar{\beta}}_{t},-\overline{\bar{\alpha}}_{t}^{\prime}, \overline{\overline{\bar{\beta}}}_{t}^{\prime}, \overline{\overline{\bar{c}}}_{t}, \bar{B}_{0},-\rho_{e}^{0}, \text { and } \rho_{m}^{0},
$$

respectively, replace the original parameters listed in (30b). The transducer of $(30 \mathrm{a})\left(c_{3}=1\right)$ converts to the transducer of (37) $\left(c_{3}=-1\right)$, and vice versa, by reversing the signs of the off-diagonal elements in the third row and column of $L^{\prime \prime}$.

When the transposed parameters (37) assume the values of the original parameters (30b), the operator $L^{-}$and its adjoint $L^{-"}$ become identical to form a self-adjoint operator for which the adjoint and original transducer are one and the same. In that case, $\rho_{e}^{0}$ must be zero $\left(\rho_{e}^{0}=-\rho_{e}^{0}\right)$, which corresponds to the magnetic-type coupling of Primakoff and Foldy [7].

The equations represented by the operators $L$ and $L^{-}$describe the same transducer. However, the equations represented by the adjoints $L^{\prime \prime}$ and $L^{-"}$ refer, in general, to different adjoint transducers. Section 5 shows that a transducer self-adjoint with respect to $L$ satisfies reciprocity, while a transducer self-adjoint with respect to $L^{-}$satisfies antireciprocity.

\section{Generalized Reciprocity Relations}

The derivation of the desired reciprocity relations can now be accomplished with the help of the reciprocity lemma (36). It will also be demonstrated that the SIM relations (9) and PWSM eqs (8) result as a by-product of the reciprocity derivation. Such a demonstration is included simply to prove that the two matrix descriptions of a transducer, which satisfies eqs (12-19), need not be postulated as a definition of linearity, but follow from the linearity of (12-19). 
The reciprocity lemma (36) for adjoint transducers may be written as

$$
\int_{A_{0}}\left[\left(\bar{E}^{\prime \prime} \times \bar{H}\right)-\left(\bar{E} \times \bar{H}^{\prime \prime}\right)\right] \cdot \hat{n} d a \pm \int_{A_{0}}\left(p^{\prime \prime} u_{n}-p u_{n}^{\prime \prime}\right) d a=0,
$$

where the plus sign refers to the operator $L$ and the minus $\operatorname{sign}$ to $L^{-}$. The surface $A_{0}$ of the transducer has been taken in the surrounding fluid, so that the stress tensor relates to pressure like

$$
\begin{aligned}
& -\hat{n} \cdot \overline{\bar{T}} \cdot \hat{n}=p \\
& -\hat{n} \cdot \overline{\bar{T}}^{\prime \prime} \cdot \hat{n}=p^{\prime \prime},
\end{aligned}
$$

except on the feed area $S_{0}$ where the contribution to the integral is assumed negligible.

The integral over $A_{0}$ of the em fields expands to

$$
V_{0}^{\prime \prime} I_{0}-V_{0} I_{0}^{\prime \prime}+\int_{A_{0}-S_{0}}\left[\left(\bar{E}^{\prime \prime} \times \bar{H}\right)-\left(\bar{E} \times \bar{H}^{\prime \prime}\right)\right] \cdot \hat{n} d a
$$

( $\hat{n}$ points into the transducer), after substitutions of the modal fields normalized to unity by (7). The contribution from the integral over $A_{0}-S_{0}$, the area of the transducer excluding the area $S_{0}$, vanishes if either of the following conditions is satisfied:

(1) The surface $A_{0}-S_{0}$ of the transducer (but not necessarily the surface $A_{p}-S_{0}$ of the power supply) is electromagnetically shielded so that $\bar{E}, \bar{E}^{\prime \prime}$ or $\bar{H}, \bar{H}^{\prime \prime}$ are zero on $A_{0}-S_{0}$. (Prescribed boundary conditions on the adjoint transducer are chosen to follow those of the original transducer.)

(2) The surface $A_{p}-S_{0}$ of the power supply (but not necessarily the surface $A_{0}-S_{0}$ of the transducer) is electromagnetically shielded and no em sources exist external to the transducerplus-(power supply or detector) A. (Regions of linear em material are permitted outside $A$.) Under these conditions, the surface $A_{0}-S_{0}$ may be enlarged to a surface of infinite radius where the Sommerfeld radiation condition or the exponential decay in electromagnetically lossy media demands that the integral be zero.

If the transducer operates at quasi-static frequencies so that the enı fields outside the transducer obey

$$
\begin{aligned}
& \bar{E}=-\nabla \psi, \bar{E}^{\prime \prime}=-\nabla \psi^{\prime \prime} \\
& \nabla \times \bar{H}=\bar{J}, \nabla \times \bar{H}^{\prime \prime}=\bar{J}^{\prime \prime},
\end{aligned}
$$

modal theory may not apply, but substitution of (40) into the em integral of (38) shows that the contribution over $A_{0}-S_{0}$ still vanishes. That is, no shielding is required at quasi-static frequencies of operation for the expression

to represent the em term of (38).

$$
V_{0}^{\prime \prime} I_{0}-V_{0} I_{0}^{\prime \prime}
$$

With the em integral over $A_{0}-S_{0}$ zero, (38) becomes

$$
V_{0}^{\prime \prime} I_{0}-V_{0} I_{0}^{\prime \prime} \pm \int_{A_{0}}\left(p^{a} u_{n}-p u_{n}^{a}\right) d a=0 .
$$

Because the fields of the transducer and its adjoint exist ii lependently and the velocity-current variables of each transducer may assume arbitrary values, (41) transforms into the SIM relations by first choosing

which gives

$$
I_{01}^{\prime \prime}=1, \quad u_{n 1}^{\prime \prime}(\bar{r})=0,
$$

$$
V_{0}=V_{01}^{u} I_{0} \pm \int_{A_{0}} p_{1}^{a}(\bar{r}) u_{n}(\bar{r}) d a
$$

then choosing

$$
I_{02}^{u}=0, u_{n 2}^{u}(\bar{r})=\delta\left(\bar{r}-\bar{r}^{\prime}\right),
$$


which gives

$$
p(\bar{r})= \pm V_{02}^{\prime \prime}(\bar{r}) I_{0}+\int_{A_{0}} p_{2}^{\prime \prime}\left(\bar{r}, \bar{r}_{0}\right) u_{n}\left(\bar{r}_{0}\right) d a_{0} .
$$

Comparison of (43) with (9) shows that the various spatial impedances equate as

$$
\begin{aligned}
Z_{b} & =V_{01}^{\prime \prime}, & h^{\prime}(\bar{r}) & = \pm p_{1}^{\prime \prime}(\bar{r}), \\
h(\bar{r}) & = \pm V_{02}^{\prime \prime}(\bar{r}), & Z_{0}\left(\bar{r}, \bar{r}_{0}\right) & =p_{2}^{\prime \prime}\left(\bar{r}, \bar{r}_{0}\right) .
\end{aligned}
$$

The PWSM equations (8) are disclosed in a similar manner once $(2,3,6)$ and the acoustical reciprocity theorem,

$$
\int_{1}\left(p^{1} u_{n}^{2}-p^{2} u_{n}^{1}\right) d a+\int_{S_{1}}\left(p^{1} u_{z}^{2}-p^{2} u_{z}^{1}\right) d \bar{R}=0
$$

as applied to any two acoustic fields supported by the fluid between $A$ and $S_{1}$, convert (4l) to

$$
\eta_{0}\left(b_{0} a_{0}^{\prime \prime}-b_{0}^{\prime \prime} a_{0}\right) \pm \int_{\bar{K}} \eta(K)\left[a(\bar{K}) b^{\prime \prime}(-\bar{K})-a^{\prime \prime}(-\bar{K}) b(\bar{K})\right] d \bar{K}=0 .
$$

Since the values of $a_{0}, a$ and $a_{0}^{\prime \prime}, a^{\prime \prime}$ may be designated arbitrarily and independently of each other, choose

and

$$
a_{01}^{\prime \prime}=1, a_{1}^{\prime \prime}(\bar{K})=0,
$$

$$
a_{02}^{\prime \prime}=0, a_{2}^{\prime \prime}(\bar{K})=\delta(\bar{K}+\bar{L}) \text {, }
$$

to extract the PWSM equations from (46),

$$
\begin{gathered}
b_{0}=b_{01}^{\prime \prime} a_{0} \mp \frac{1}{\eta_{0}} \int_{\bar{L}} \eta(L) b_{1}^{a}(-\bar{L}) a(\bar{L}) d \bar{L} \\
b(\bar{K})=\mp \frac{\eta_{0}}{\eta(K)} b_{02}^{\prime \prime}(\bar{K}) a_{0}+\frac{1}{\eta(K)} \int_{L} \eta(L) b_{2}^{\prime \prime}(\bar{K},-\bar{L}) a(\bar{L}) d \bar{L} .
\end{gathered}
$$

Equations (48) coincide with (8) under the replacements,

$$
\begin{gathered}
S_{00}=b_{01}^{\prime \prime}, S_{01}(\bar{L})=\mp \eta(L) b_{1}^{\prime \prime}(-\bar{L}) / \eta_{0}, \\
S_{10}(\bar{K})=\mp \eta_{0} b_{02}^{\prime \prime}(\bar{K}) / \eta(K), S_{11}=\eta_{(}(L) b_{2}^{\prime \prime}(\bar{K},-\bar{L}) / \eta(K) .
\end{gathered}
$$

If the conditions of (42) and (47) are applied to the given transducer rather than its adjoint transducers, (41) and (46) produce (43) and (48) with the given transducer and adjoint transducers interchanged. That is, the SIM relations and the PWSM equations describe the adjoint transducers as well as the original transducer.

The generalized reciprocity relations between a given transducer and its adjoint transducers may also be extracted from (41) and (46) by eliminating the pressures and voltages of (41) with the SIM relations, and the outgoing amplitudes of (46) with the PWSM equations. Such a procedure yields 


$$
\begin{aligned}
I_{0}^{\prime \prime}\left(Z_{b}^{\prime \prime}-Z_{b}\right) I_{0}+I_{0} \int_{A_{0}}\left[h^{\prime \prime}(\bar{r}) \mp h(\bar{r}) \mid u_{n}^{\prime \prime}(\bar{r}) d a\right. & -I_{0}^{\prime \prime} \int_{A_{0}}\left[h^{\prime}(\bar{r}) \mp h^{\prime \prime}(\bar{r}) \mid u_{n}(\bar{r}) d a\right. \\
& \pm \int_{I_{0}} \int_{A_{0}} u_{n}^{\prime \prime}(\bar{r})\left[Z_{0}^{\prime \prime}\left(\bar{r}_{0}, \bar{r}\right)-Z_{0}\left(\bar{r}, \bar{r}_{0}\right) \mid u_{n}\left(\bar{r}_{0}\right) d a_{0} d a=0\right.
\end{aligned}
$$

for (41), and

$$
\begin{aligned}
\eta_{01} a_{0}^{\prime \prime}\left(S_{00}^{\prime \prime}-S_{00}\right) a_{00} & +a_{00} \int_{\bar{L}}\left[\eta_{00} S_{01}^{\prime \prime}(\bar{L}) \mp \eta(L) S_{10}(-\bar{L})\right] a^{\prime \prime}(\bar{L}) d \bar{L} \\
& +a_{0}^{\prime \prime} \int_{\bar{L}}\left[\eta_{00} S_{01}(\bar{L}) \mp \eta(L) S_{10}^{\prime \prime}(-\bar{L})\right] a(\bar{L}) d \bar{L} \\
& \pm \int_{\bar{L}} \int_{\bar{K}} a^{\prime \prime}(\bar{L})\left[\eta(K) S_{11}^{\prime \prime}(-\bar{K}, \bar{L})-\eta(L) S_{11}(-\bar{L}, \bar{K})\right] a(\bar{K}) d \bar{K} d \bar{L}=0
\end{aligned}
$$

for (46).

Since they must hold for arbitrary values of the inputs, (50) and (51) imply

$$
\begin{gathered}
Z_{b}^{\prime \prime}=Z_{b} \\
h^{\prime \prime}(\bar{r})= \pm h(\bar{r}) \\
h^{\prime \prime}(\bar{r})= \pm h^{\prime}(\bar{r}) \\
Z_{0}^{\prime \prime}\left(\bar{r}_{0}, \bar{r}\right)=Z_{0}\left(\bar{r}, \bar{r}_{0}\right)
\end{gathered}
$$

and

$$
\begin{gathered}
S_{00}^{\prime \prime}=S_{00} \\
\eta_{0} S_{01}^{\prime \prime}(\bar{K})= \pm \eta(K) S_{10}(-\bar{K}) \\
\eta(K) S_{10}^{\prime \prime}(\bar{K})= \pm \eta_{0} S_{01}(-\bar{K}) \\
\eta(K) S_{11}^{\prime \prime}(-\bar{K}, \bar{L})=\eta(L) S_{11}(-\bar{L}, \bar{K}) .
\end{gathered}
$$

Either the " $b$ " or " $c$ " equation of (52) and (53) may be considered redundant since the entire derivation could be repeated with the given and adjoint transducers interchanged.

It is (53b) that enables the characteristic receiving matrix $S_{01}$ of an arbitrary linear transducer to be expressed mathematically as a function of the equivalent sources within the adjoint transducers [19]. As mentioned in the introduction, such expressions have special utility in the extrapolation techniques of the PWSM formulation $[4,8]$.

When a transducer is self-adjoint with respect to $L$ or $L^{-},(52)$ and (53) reduce to the original reciprocity relations of (10) and (11). Reciprocity defined by both Kerns and Foldy-Primakoff is satisfied by an electroacoustic transducer that exhibits electric-type coupling (e.g., the condenser microphone) to form a self-adjoint operator $L$. Antireciprocity is satisfied by a transducer that exhibits magnetic-type coupling (e.g., the dynamic speaker) to form a self-adjoint operator $L^{-}$. If the static fields as well as the piezoelectric and piezomagnetic coupling coefficients vanish, comparison of (30a) with (37) reveals that both adjoint transducers become identical to the original transducer, and by (52) and (53), the transmitting and receiving parameters $h, h^{\prime}, S_{10}, S_{01}$ also vanish. In other words, there would be no electroacoustic coupling-a result that the constitutive relations (14-18) confirm. 


\section{Adjoint Reciprocity Theorems}

The generalized reciprocity relations (52) and (53) may find expression in a variety of derivative "reciprocity theorems and principles." As a consequence of (52) or (53), the "electroacoustic reciprocity theorem" $[6,20]$ for reciprocal transducers extends to nonreciprocal transducers by the relating of the microphone response of the given transducer to the speaker response of its adjoint transducers (and vice versa). Similarly, the "principle of reciprocity" [21] that relates scattered pressure at a point $B$ from a point source at $A$ to scattered pressure at point $A$ when the point source is moved to $B$ may be extended to nonreciprocal transducers through the concept of the adjoint transducer. The following derivation of the aforementioned results are performed easily and in great generality by using the Kerns PWSM description.

The standard speaker response $S(\bar{r})$ of a given transducer is defined as the ratio of the pressure at a point $r$ in the ambient fluid to the transducer input current $I_{0}[6]$,

$$
S(\bar{r}) \equiv \frac{p(\bar{r})}{I_{0}} .
$$

Because incoming acoustic waves are assumed zero, i.e., $a(K)=0$, the pressure in (2a) may be expressed in terms of $b(K)=V(K)$ alone,

$$
p(\bar{r})=\frac{1}{2 \pi} \int_{\bar{K}} b(\bar{K}) e^{i \gamma z} e^{i \bar{K} \cdot \bar{r}} d \bar{K} .
$$

The factor $e^{i \gamma z}$ appearing in the integral of (55) allows the $z$-coordinate of $\bar{r}$ to assume values other than zero. (The fluid must contain no sources or inhomogeneities between $z=0$ and the $z$ in (55).) The PWSM equations (8), which reduce to

$$
\begin{gathered}
b_{0}=S_{00} a_{0} \\
b(\bar{K})=S_{10}(\bar{K}) a_{0}
\end{gathered}
$$

when $a(\bar{K})=0$, combine with $(6 \mathrm{~b})$ to yield the following expression for $b(\bar{K})$ in terms of $I_{o}$ :

$$
b(\bar{K})=\frac{S_{10}(\bar{K}) I_{0}}{\left(1-S_{00}\right) \eta_{0}} .
$$

After substitution of $b(\bar{K})$ from (57) into (55), the speaker response (54) takes the final form of

$$
S(\bar{r})=\frac{1}{2 \pi\left(1-S_{00}\right) \eta_{0}} \int_{\bar{K}} S_{10}(\bar{K}) e^{i \bar{k} \cdot \bar{r}} d \bar{K}, \bar{k}=\bar{K}+\gamma \hat{e}_{z}
$$

The adjoint microphone response is defined as the ratio of the open-circuit voltage $V_{0}$ for an adjoint transducer in the presence of a spherical pressure wave centered at point $\bar{r}$ in the fluid to the pressure in the spherical wave at a reference point $\bar{r}_{c}[6]$,

$$
\begin{gathered}
M^{\prime \prime}(\bar{r}) \equiv \frac{V_{0}}{p_{r}\left(\bar{r}_{c}\right)}, \\
p_{r}\left(\bar{r}_{c}\right)=\frac{\mathscr{A} e^{i k}\left|\bar{r}_{c}-\bar{r}\right|}{\left|\bar{r}_{c}-\bar{r}\right|} .
\end{gathered}
$$


(The amplitude of the spherical wave is denoted by $\mathscr{A}$.) Since $I_{0}$ is zero, $a_{0}$ equals $b_{0}$, and $V_{0}$ is equal to $2 a_{0}$. The scattering-matrix equation (8a) for an adjoint transducer relates $a_{0}$ to $a(\bar{K})$,

$$
a_{0}=\frac{1}{\left(1-S_{00}^{\prime \prime}\right)} \int_{\bar{K}} S_{01}^{\prime \prime}(\bar{K}) a(\bar{K}) d \bar{K}
$$

The incoming amplitudes $a(\bar{K})$ are given by $(4 \mathrm{a})$ as

$$
a(\bar{K})=V(\bar{K})=\frac{1}{2 \pi} \int_{S_{1}} p_{r}\left(\bar{R}_{c}\right) e^{-i \bar{K}^{\prime} \cdot \bar{R}_{r}} d \bar{R}_{c} .
$$

Replacement of the pressure in (62) by its value given in (60) enables the explicit determination of $a(\bar{K})$,

$$
a(\bar{K})=\frac{\mathscr{A} i}{\gamma} e^{i \gamma z} e^{-i \bar{K} \cdot \bar{R}},
$$

which combines with (61), (60), and (59) to produce the final form of the adjoint microphone response,

$$
M^{\prime \prime}(\bar{r})=\frac{2 i d}{\left(1-S_{00}^{\prime \prime}\right) e^{i k d}} \int_{\bar{K}} \frac{S_{01}^{\prime \prime}(-\bar{K})}{\gamma} e^{i \bar{k} \cdot \bar{r}} d \bar{K}, d=\left|\bar{r}_{c}-\bar{r}\right| .
$$

Since the adjoint reciprocity relations require that

$$
\begin{gathered}
S_{00}^{\prime \prime}=S_{00} \\
\eta_{0} S_{01}^{\prime \prime}(-\bar{K})=\frac{ \pm \gamma}{\omega \rho_{0}} S_{10}(\bar{K}),
\end{gathered}
$$

(58) and (64) show that

$$
\left|\frac{M^{\prime \prime}(\bar{r})}{S(\bar{r})}\right|=\frac{4 \pi d}{\omega \rho_{0}}
$$

or that the magnitude of the ratio of microphone response at $\bar{r}$ of the adjoint transducers to the speaker response at $\bar{r}$ of the original transducer is a constant independent of the position $\bar{r}$ and of the particular transducer involved. The constant could also be made independent of the distance $d$ to the reference point $\bar{r}_{c}$ if the microphone response were "normalized" to the amplitude $\mathscr{A}$ of the spherical wave. If the transducer is self-adjoint (reciprocal or antireciprocal) (65) becomes identical to the standard electroacoustic reciprocity theorem [20].

Next, consider the derivation of the adjoint "principle of reciprocity" which applies to the scattering of a spherical wave from a given scatterer (or transducer terminated in a passive load). The scattered pressure $p_{s c}\left(\bar{r}_{B}, \bar{r}_{1}\right)$ at a point $\bar{r}_{B}$ in the fluid caused by a spherical wave centered at a point $\bar{r}_{A}$ in the fluid is given, as in (55), by

$$
p_{s c}\left(\bar{r}_{B}, \bar{r}_{A}\right)=\frac{1}{2 \pi} \int_{\bar{K}} b(\bar{K}) e^{i \bar{k} \cdot \bar{r}} d \bar{K}
$$


where $b(\bar{K})$ may be found in terms of a $(\bar{K})$ from the scattering equations (8):

$$
b(\bar{K})=\int_{\bar{L}}\left(\frac{S_{10}(\bar{K}) S_{01}(\bar{L}) \Gamma_{T}}{\left(1-\Gamma_{T} S_{00}\right)}+S_{11}(\bar{K}, \bar{L})\right) a(\bar{L}) d \bar{L} .
$$

The reflection coefficient $\Gamma_{T}$ is related to the terminating load $Z_{T}=-V_{0} / I_{0}$ by

$$
\Gamma_{T}=\frac{Z_{T} \eta_{0}-1}{Z_{T} \eta_{0}+1}
$$

Since the source is a spherical pressure wave centered at $\bar{r}_{A}$, the amplitudes a $(\bar{L})$ take the same form as in (63), i.e.,

$$
a(\bar{L})=\frac{\mathscr{A} i}{\gamma(L)} e^{i \gamma(L) z_{i}} e^{-i \bar{L} \cdot \bar{R}_{A}}
$$

Substitution of (69) and (67) into (66) results in the scattered pressure

$$
p_{s c}\left(\bar{r}_{b}, \bar{r}_{A}\right)=\frac{\mathscr{A} i}{2 \pi} \int_{\bar{K}} \int_{\bar{L}} \frac{\left(\frac{\left.S_{10}(\bar{K}) S_{01}(-\bar{L}) \Gamma_{T}\right)}{1-\Gamma_{T} S_{00}}+S_{11}(\bar{K},-\bar{L})\right)}{\gamma(L)} e^{i \bar{k} \cdot \bar{r}_{B}} e^{i \bar{l} \cdot \bar{r}_{A}} d \bar{L} d \bar{K}
$$

If the position of the source and observer is interchanged and the given transducer is replaced by an adjoint transducer, the scattered pressure at $\bar{r}_{A}$ is found in a similar manner to be

$$
p_{s c}^{a}\left(\bar{r}_{A}, \bar{r}_{B}\right)=\frac{\mathscr{A} i}{2 \pi} \int_{\bar{K}} \int_{\bar{L}} \frac{\left(\frac{\left.\mathrm{S}_{10}^{a}(\bar{K}) S_{01}^{a}(-\bar{L}) \Gamma_{T}\right)}{1-\Gamma_{T} S_{00}^{a}}+S_{11}^{a}(\bar{K},-\bar{L})\right)}{\gamma(L)} e^{i \bar{k} \cdot \bar{r}_{A}} e^{i \bar{l} \cdot \bar{r}_{B}} d \bar{L} d \bar{K}
$$

(The termination for the adjoint transducers is kept at $Z_{T}$.)

The adjoint reciprocity relations (53) demonstrate from (70) and (71) that

$$
p_{s c}\left(\bar{r}_{B}, \bar{r}_{A}\right)=p_{s c}^{a}\left(\bar{r}_{A}, \bar{r}_{B}\right),
$$

which completes the proof of the adjoint principle of reciprocity. (Note that the bracketed quantity in the integrands of (70) and (71) represents an equivalent scattering matrix with reciprocal properties identical to $S_{11}$.) That is, the scattered pressure measured at a point $\bar{r}_{B}$ from a point source at $\bar{r}_{A}$ is equal to the scattered pressure measured at the point $\bar{r}_{A}$ when the point source is moved to $\bar{r}_{B}$ and an adjoint transducer replaces the given transducer. If the transducer is self-adjoint (reciprocal or antireciprocal) (72) expresses the usual principle of reciprocity [21] for scatterers.

\section{Power Relations}

In this section power relations are developed and used to show that the dissipative characteristics (lossiness, losslessness, and "gaininess") of the material of the given transducer are preserved pointwise in the adjoint transducers. Such a result lends support to the feasibility of producing the adjoint transducers in the laboratory. 
For harmonic fields, the time-average em and acoustic power flow per unit area is given by $\frac{1}{2} \operatorname{Re}\left[\bar{E} \times \bar{H}^{*}\right]$ and $-\frac{1}{2} \operatorname{Re}\left[\overline{\bar{T}} \cdot \bar{u}^{*}\right]$ respectively. (The asterisk denotes the complex conjugate.) The total time-average power $P$ flowing into the electroacoustic transducer through its surface $A_{0}$ is, therefore, given by

$$
P=\frac{1}{2} \operatorname{Re} \oint_{t_{0}}\left[\bar{E} \times \bar{H}^{*}-\overline{\bar{T}} \cdot \bar{u}^{*}\right] \cdot \hat{n} d a
$$

where $\hat{n}$ denotes the unit normal into the transducer. Since the surface $A_{0}$ lies in or on the boundary of the ambient fluid,

$$
\hat{n} \cdot \overline{\bar{T}} \cdot \bar{u}^{*}=-p u_{n}^{*}
$$

and the power equation (73) becomes

$$
P=\frac{1}{2} \operatorname{Re} \oint_{A_{0}}\left[\left(\bar{E} \times \bar{H}^{*}\right) \cdot \hat{n}+p u_{n}^{*}\right] d a
$$

If the transducer is electromagnetically shielded, the em part of the integral (74) evaluates simply as $V_{0} I_{0}^{*}$ by expanding $\bar{E}$ and $\bar{H}^{*}$ in terms of the waveguide mode, i.e., from (5)

$$
\bar{E}_{t}=V_{0} \bar{e}_{0}, \bar{H}_{t}^{*}=I_{0}^{*} \bar{h}_{0},
$$

and making use of the normalization equation (7). (Waveguide theory shows that the basis fields $\left(\bar{e}_{0}, \bar{h}_{0}\right)$ of a propagating mode in a uniform, isotropic guide may be chosen real.)

The SIM relations (9) convert the power expression (74) to an Hermitian form containing continuous $\left(u_{n}(\bar{r})\right)$ and discrete $\left(I_{0}\right)$ variables:

$$
\begin{aligned}
P & =I_{0}\left(Z_{b}+Z_{b}^{*}\right)+\int_{A_{0}}\left[I_{0}\left(h^{\prime *}(\bar{r})+h(\bar{r})\right) u_{n}^{*}(\bar{r})+I_{0}^{*}\left(h^{\prime}(\bar{r})+h^{*}(\bar{r})\right) u_{n}(\bar{r})\right] d a \\
& +\int_{A_{0}} \int_{A_{0}} u_{n}\left(\bar{r}_{0}\right)\left[Z_{0}\left(\bar{r}, \bar{r}_{0}\right)+Z_{0}^{*}\left(\bar{r}_{0}, \bar{r}\right)\right] u_{n}^{*}(\bar{r}) d a_{0} d a .
\end{aligned}
$$

(The taking of the real part has been accomplished by adding each term to its complex conjugate.)

A transducer may be classified as lossy, lossless, or "gainy" if, for all values of $u_{n}$ and $I_{0}$ (except $u_{n}, I_{0}$ all zero), the power $P$ is positive, zero, or negative, i.e., if the Hermitian form (75) is positive definite, zero, or negative definite [22].

Mercer's theorem [23], which can be generalized to provide necessary and sufficient conditions on the spacial impedances $Z_{b}, h, h^{\prime}$, and $Z_{0}$ for the power $P$ to belong to one of the "definite" value classes, reduces to very simple relationships among the spacial impedances when the transducer is lossless. In the lossless case, i.e., when the power expression (75) is identically zero for all values of $u_{n}$ and $I_{0}$, various combinations of $I_{0}$ equal to zero and $u_{n}(\bar{r})$ equal to zero or a sum of delta functions can be chosen to prove that the spatial impedances satisfy the following equalities:

$$
\begin{aligned}
Z_{b}+Z_{b}^{*} & =0 \quad \text { (i.e., } Z_{b} \text { is imaginary) } \\
h^{\prime}(\bar{r})+h^{*}(\bar{r}) & =0 \\
Z_{0}\left(\bar{r}, \bar{r}_{0}\right)+Z_{0}^{*}\left(\bar{r}_{0}, \bar{r}\right) & =0 .
\end{aligned}
$$


A reciprocal or antireciprocal transducer also satisfies (11), which combine with (76) to show that $Z_{0}$ (as well as $Z_{b}$ ) is imaginary, and that both $h, h^{\prime}$ are imaginary or real depending upon whether the transducer is reciprocal or antireciprocal respectively. It can be demonstrated [9] that no simple relations similar to (76) exist among the scattering matrices of lossless transducers.

Substitution of the adjoint relations (52) for the spacial impedances into (75) yields an expression (similar to (75)) which can be manipulated slightly to prove that the power input to both adjoint transducers (one associated with $L$, the other with $L^{-}$) belong to the same value class, i.e., have the same definite or indefinite form, as the power input to the original transducer. However, the power input to the adjoint transducers does not, necessarily remain the same as the power input to the original transducer for given excitation $I_{0}$ and $u_{n}(\bar{r})$.

Finally, consider the problem of expressing the power relation (73) in terms of the internal fields and material parameters of the electroacoustic transducer. The divergence theorem transforms the surface integral (73) into a volume integral over the volume $\mathscr{V}_{0}$ (enclosed by $S_{0}$ ) of the transducer to produce,

$$
\begin{gathered}
P=\int_{\gamma_{0}}\left[\bar{H}^{*} \cdot(\nabla \times E)+\bar{H} \cdot\left(\nabla \times \bar{E}^{*}\right)-\bar{E} \cdot\left(\nabla \times \bar{H}^{*}\right)-\bar{E}^{*} \cdot(\nabla \times \bar{H})\right. \\
\left.-(\nabla \cdot \overline{\bar{T}}) \cdot \bar{u}^{*}-\left(\nabla \cdot \overline{\bar{T}}{ }^{*}\right) \cdot \bar{u}-\overline{\bar{T}}: \nabla \bar{u}^{*}-\overline{\bar{T}}^{*}: \nabla \bar{u}\right] d V,
\end{gathered}
$$

after the divergence is expanded, and the taking of the real part is accomplished by adding each term to its complex conjugate. The equations (12-19) allows the integrand $P_{v}$ of the power integral (77) to be written as an Hermitian form,

$$
\left[\bar{H}^{*}, \bar{E}^{*}, \bar{s}^{*}, \bar{u}^{*}\right]\left(\begin{array}{cccc}
i \omega\left(\overline{\bar{\mu}}-\overline{\bar{\mu}}_{t}^{*}\right) & i \omega\left(\nu-\overline{\bar{\tau}}_{t}^{*}\right) & i \omega\left(\overline{\bar{\beta}}-\overline{\bar{\beta}}_{t}^{*}\right) & 0 \\
i \omega\left(\overline{\bar{\tau}}-\overline{\bar{\nu}}_{t}^{*}\right) & {\left[i \omega\left(\overline{\bar{\epsilon}}-\overline{\bar{\epsilon}}_{t}^{*}\right)-\left(\overline{\bar{\sigma}}+\overline{\bar{\sigma}}_{t}^{*}\right)\right] i \omega\left(\overline{\bar{\alpha}}-\overline{\bar{\alpha}}_{t}^{\prime}\right)} & \bar{B}_{0} \times\left(\overline{\bar{\sigma}}+\overline{\bar{\sigma}}_{t}^{*}\right) \\
i \omega\left(\overline{\bar{\beta}}^{\prime}-\overline{\bar{\beta}}^{*}\right) & i \omega\left(\overline{\bar{\alpha}}-\overline{\bar{\alpha}}_{t}^{*}\right) & -i \omega\left(\overline{\bar{c}}-\overline{\bar{c}}_{t}^{*}\right) & 0 \\
0 & -\bar{B}_{0} \times\left(\overline{\bar{\sigma}}+\overline{\bar{\sigma}}_{t}^{*}\right) & 0 & \bar{B}_{0} \times\left(\overline{\bar{\sigma}}+\overline{\bar{\sigma}}_{t}^{*}\right) \times \bar{B}_{0}
\end{array}\right)\left(\begin{array}{c}
\bar{H} \\
\bar{E} \\
\overline{\bar{s}} \\
\bar{u}
\end{array}\right)
$$

or simply

$$
P_{r}=\tilde{X} * \mathscr{H} X
$$

$P_{v}$ may be interpreted as power input per unit volume of the transducer.

A sufficient condition for the total time-average power input to be positive definite, zero, or negative definite is for the Hermitian integrand (78) to be correspondingly positive definite, zero, or negative definite. Likewise, a standard theorem of linear algebra [22] states that the matrix $\mathscr{H}$ must belong to the same value class as the associated Hermitian form (78), provided $\bar{H}, \bar{E}$, $\overline{\bar{s}}$ and $\bar{u}$ may assume arbitrary values at a point. In particular, if $P_{v}$ is zero, the transducer is lossless, $\mathscr{H}$ must be zero and therefore the material parameters satisfy the following simple relationships:

$$
\overline{\bar{\sigma}}=-\overline{\bar{\sigma}}_{t}^{*}, \overline{\bar{\epsilon}}=\overline{\bar{\epsilon}}_{t}^{*}, \overline{\bar{\mu}}=\overline{\bar{\mu}}_{t}^{*}, \overline{\bar{\nu}}=\overline{\bar{\tau}}_{t}^{*}, \overline{\overline{\underline{ }}}=\overline{\bar{p}}_{t}^{*}, \alpha^{\prime}=\overline{\bar{\alpha}}_{t}^{*}, \text { and } \overline{\bar{\beta}}^{\prime}=\overline{\bar{\beta}}_{t}^{*} .
$$

If, in addition, the transducer is reciprocal or antireciprocal, (30) and (37) show that the material parameters also satisfy

$$
\overline{\bar{\sigma}}=\overline{\bar{\sigma}}_{t}, \overline{\bar{\epsilon}}=\overline{\bar{\epsilon}}_{t}, \overline{\bar{\mu}}=\overline{\bar{\mu}}_{t}, \overline{\bar{\nu}}=\overline{\bar{\tau}}_{t}, \overline{\overline{\underline{y}}}=\overline{\bar{c}}_{t}, \overline{\bar{\alpha}}^{\prime}= \pm \overline{\bar{\alpha}}_{t}, \text { and } \overline{\bar{\beta}}^{\prime}=\mp \overline{\bar{\beta}}_{t}^{*},
$$

where the upper and lower sign in the last two equations of (81) refer to reciprocal and antireciprocal transducers respectively. Equations (80) and (81) together imply that a lossless (in the sense of (80)) reciprocal or antireciprocal transducer possesses real $\overline{\bar{\sigma}}, \overline{\bar{\epsilon}}, \overline{\bar{\mu}}, \overline{\bar{c}}$, and imaginary $\overline{\bar{\nu}}$ and $\overline{\bar{\tau}}$. The 
piezoelectric triadics $\overline{\bar{\alpha}}, \overline{\bar{\alpha}}^{\prime}$ are real for reciprocal and imaginary for antireciprocal lossless transducers. Conversely, the piezomagnetic triadics $\overline{\bar{\beta}}, \overline{\bar{\beta}}^{\prime}$ are imaginary for reciprocal and real for antireciprocal lossless transducers.

Equations (30) and (37) may also be used to demonstrate from (78) and (77) that the total power input for the two transducers adjoint to the original transducer belong to the same value class or have the same dissipative character as the original transducer-a result which concurs with the previous analysis on the spacial impedance power relation (75).

\section{Concluding Remarks}

A brief summary of the main properties of the $L$ and $L^{-}$adjoint transducers is displayed in tables 1-4. From an experimental standpoint, it is naturally of interest to know if the adjoint transducers are physically realizable, i.e., producible in the laboratory. For example, if a transducer adjoint to a given transducer could be produced, the receiving characteristics $\left(S_{01}\right)$ of the given transducer could be determined by measuring the transmitting characteristics $\left(S_{10}^{\prime \prime}\right)$ of the adjoint transducer. Such an indirect experimental procedure would be desirable with a set-up designed to measure transducer far-field patterns.

TABLE 1.

\begin{tabular}{|c|c|c|c|}
\hline \multicolumn{2}{|l|}{$\begin{array}{c}\text { Given transducer } \\
\text { (described by eqs }(12-19) \text { ) }\end{array}$} & $L$ Adjoint transducer & $L^{-}$Adjoint transducer \\
\hline \multicolumn{2}{|l|}{ Internal Characteristics } & Internal characteristics & Internal characteristics \\
\hline Conductivity & $\overline{\bar{\sigma}}$ & $\overline{\bar{\sigma}}^{a}=\overline{\bar{\sigma}}_{t}$ & $\overline{\bar{\sigma}}^{a}=\overline{\bar{\sigma}}_{t}$ \\
\hline Permittivity & $\overline{\bar{\epsilon}}$ & $\overline{\overline{\epsilon^{a}}}=\overline{\overline{\epsilon_{t}}}$ & $\overline{\bar{\epsilon}}^{a}=\overline{\overline{\epsilon_{t}}}$ \\
\hline Permeability & $\overline{\bar{\mu}}$ & $\overline{\bar{\mu}}^{a}=\overline{\bar{\mu}}_{t}$ & $\overline{\bar{\mu}}^{a}=\overline{\bar{\mu}}_{t}$ \\
\hline Hooke's tensor & $\underline{\underline{\underline{\underline{T}}}}$ & $\overline{\overline{\bar{c}}}=\overline{\overline{\overline{\bar{i}}}}$ & $\overline{\underline{\underline{\underline{c}}}}^{a}=\overline{\overline{\overline{\bar{c}}}}$ \\
\hline Magneto-electric tensors & $\overline{\bar{\nu}}, \overline{\bar{\tau}}$ & $\overline{\bar{\nu}}^{a}=-\overline{\bar{\tau}}_{t}, \overline{\bar{\tau}}^{a}=-\overline{\bar{\nu}}_{t}$ & $\overline{\bar{\nu}}^{a}=-\overline{\bar{\tau}}_{t}, \overline{\bar{\tau}}^{a}=-\overline{\bar{\nu}}_{t}$ \\
\hline $\begin{array}{l}\text { Direct peizoelectric and piezomag- } \\
\text { netic tensors }\end{array}$ & $\underset{\alpha, \beta}{\equiv}$ & $\overline{\bar{\alpha}}^{a}=\overline{\bar{\alpha}}_{t}^{\prime}, \overline{\overline{\beta^{a}}}=-\overline{\bar{\beta}}$ & $\overline{\bar{\alpha}}^{\alpha}=-\alpha_{t}^{\prime}, \overline{\bar{\beta}}^{a}=\overline{\bar{\beta}}_{t}^{\prime}$ \\
\hline $\begin{array}{l}\text { Converse piezoelectric and piezo- } \\
\text { magnetic tensors }\end{array}$ & $\equiv \overline{\alpha^{\prime}}, \overline{\overline{\beta^{\prime}}}$ & 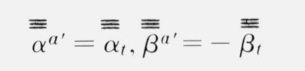 & $\bar{\equiv}_{\alpha^{a^{\prime}}=-\bar{\alpha}_{t}, \bar{\beta}^{a^{\prime}}=\overline{\bar{\beta}}_{t}}$ \\
\hline Mass density & $\rho_{m}^{0}$ & $\rho_{m}^{a 0}=\rho_{m}^{0}$ & $\rho_{m}^{a 0}=\rho_{m}^{0}$ \\
\hline Static magnetic induction & $\bar{B}_{0}$ & $\bar{B}_{0}^{a}=-\bar{B}_{0}$ & $\bar{B}_{0}^{a}=\bar{B}_{0}$ \\
\hline Static charge distribution & $\rho_{e}^{0}$ & $\rho_{e}^{a 0}=\rho_{e}^{0}$ & $\rho_{e}^{a 0}=-\rho_{e}^{0}$ \\
\hline
\end{tabular}

TABLE 2

\begin{tabular}{|c|c|c|c|}
\hline \multicolumn{2}{|c|}{$\begin{array}{c}\text { (Given transducer } \\
\text { (described by eqs }(12-19) \text { ) }\end{array}$} & $L$ Adjoint transducer & $L^{-}$Adjoint transducer \\
\hline \multicolumn{2}{|c|}{ External characteristics } & External characteristics & External characteristics \\
\hline Transmitting (PWSM) & $S_{10}(\bar{K})$ & $S_{10}^{a}(\bar{K})=S_{111}(-\bar{K}) \eta_{00} / \eta$ & $S_{10}^{n}(\bar{K})=-S_{01}(-\bar{K}) \eta_{0} / \eta$ \\
\hline Receiving (PWSM) & $S_{01}(\bar{K})$ & $S_{01}^{\prime \prime}(\bar{K})=S_{10}(-\bar{K}) \eta / \eta_{0}$ & $S_{01}^{\prime \prime}(\bar{K})-=S_{10}(-\bar{K}) \eta / \eta_{0}$ \\
\hline Acoustic scattering (PWSM) & $S_{11}(\bar{K}, \bar{L})$ & $S_{11}^{a}(\bar{K}, \bar{L})=$ & $S_{11}^{\prime \prime}(\bar{K}, \bar{L})=$ \\
\hline & & $S_{11}\left(-\bar{L}_{,}^{-}-\bar{K}\right) \eta(\bar{L}) / \eta(\bar{K})$ & $S_{11}(-\bar{L},-\bar{K}) \eta(\bar{L}) / \eta(\bar{K})$ \\
\hline EM reflection $(P W S M)$ & $S_{(0)}$ & $S_{00}^{a}=S_{00}$ & $S_{00}^{\prime \prime}=S_{00}$ \\
\hline
\end{tabular}


TABLE 2-CONTINUED

\begin{tabular}{|c|c|c|c|}
\hline \multicolumn{2}{|c|}{$\begin{array}{c}\text { Given transducer } \\
\text { (described by eqs }(12-19) \text { ) }\end{array}$} & $L$ Adjoint transducer & $L^{-}$Adjoint transducer \\
\hline \multicolumn{2}{|c|}{ External characteristics } & External characteristics & External characteristics \\
\hline Speaker transfer $(S I M)$ & $h(\bar{r})$ & $h^{\prime \prime}(\bar{r})=h^{\prime}(\bar{r})$ & $h^{a}(\bar{r})=-h^{\prime}(\bar{r})$ \\
\hline Vicrophone transfer (SIM) & $h^{\prime}(\bar{r})$ & $h^{\prime \prime^{\prime}}(\bar{r})=h(\bar{r})$ & $h^{\prime \prime}(\bar{r})=-h(\bar{r})$ \\
\hline Open-circuit acoustic (SIM) & $Z_{0}\left(\bar{r}, \bar{r}_{0}\right)$ & $Z_{00}^{\prime \prime}\left(\bar{r}, \bar{r}_{0}\right)=Z_{0}\left(\bar{r}_{0}, \bar{r}\right)$ & $Z_{0}^{a}\left(\bar{r}, \bar{r}_{0}\right)=Z_{0}\left(\bar{r}_{0}, \bar{r}\right)$ \\
\hline Blocked electrical (SIM) & $Z_{b}$ & $Z_{b}^{a}=Z_{b}$ & $Z_{\mathrm{b}}^{a}=Z_{l}$ \\
\hline
\end{tabular}

TABLE 3

\begin{tabular}{|c|c|c|c|}
\hline \multicolumn{2}{|c|}{$\begin{array}{c}\text { Civen transducer } \\
\text { (described by eqs }(12-19) \text { ) }\end{array}$} & $L$ Adjoint transducer & $L^{-}$Adjoint transducer \\
\hline \multicolumn{2}{|c|}{ P'ower input } & Power input & Power input \\
\hline Passive transducers & $\begin{array}{l}p>0 \\
p \geqslant 0 \\
p=0\end{array}$ & $\begin{array}{l}p^{\prime \prime}>0 \\
p^{\prime \prime} \geq 0 \\
p^{\prime \prime}=0\end{array}$ & $\begin{array}{l}p^{\prime \prime}>0 \\
p^{\prime \prime} \geqslant 0 \\
p^{\prime \prime}=0\end{array}$ \\
\hline Active transducers & $\begin{array}{l}p<0 \\
p \leqslant 0 \\
\text { Indefinite }\end{array}$ & $\begin{array}{l}p^{\prime \prime}<0 \\
p^{\prime \prime} \leqslant 0 \\
\text { Indefinite }\end{array}$ & $\begin{array}{l}p^{\prime \prime}<0 \\
p^{\prime \prime} \leqslant 0 \\
\text { Indefinite }\end{array}$ \\
\hline
\end{tabular}

TABLE 4

\begin{tabular}{|c|c|c|c|}
\hline \multicolumn{2}{|c|}{$\begin{array}{c}\text { Civen transducer } \\
\text { (described by eqs }(12-19))\end{array}$} & $L$ Adjoint transducer & $L^{-}$Adjoint transducer \\
\hline \multicolumn{2}{|c|}{ Adjoint reciprocity theorems } & Adjoint reciprocity theorems & Adjoint Reciprocity theorems \\
\hline Speaker Response & $S(\bar{r})$ & $S^{\prime \prime}(\bar{r})=M(\bar{r}) \omega \rho_{n} e^{i k d} / 4 \pi i d$ & $S^{\prime \prime}(\bar{r})=-M(\bar{r}) \omega \rho_{0} e^{i k d} / 4 \pi i d$ \\
\hline Microphone response & $M(\bar{r})$ & $M^{\prime \prime}(\bar{r})=S(\bar{r}) 4 \pi i d / \omega \rho_{0} e^{i k d t}$ & $M^{\prime \prime}(\bar{r})=-S(\bar{r}) 4 \pi i d / \omega \rho_{0} e^{i k d}$ \\
\hline $\begin{array}{l}\text { Scattered pressure } \\
\text { at } \bar{r}_{k} \text { from point } \\
\text { source at } \bar{r}_{1}\end{array}$ & $p_{s r}\left(\bar{r}_{B}, \bar{r}_{1}\right)$ & $p_{s c}^{\prime \prime}\left(\bar{r}_{B}, \bar{r}_{1}\right)=p_{s c}\left(\bar{r}_{1}, \bar{r}_{B}\right)$ & $p_{s c}^{\prime \prime}\left(\bar{r}_{B}, \bar{r}_{1}\right)=p_{s c}\left(\bar{r}_{A}, \bar{r}_{B}\right)$ \\
\hline
\end{tabular}

The complex problems involved in synthesizing transducer materials of prescribed electroacoustic properties is not the subject of the present paper. However, two groups of nonreciprocal transducers should be mentioned for which the creation of the adjoint transducers require, in principle, a relatively simple procedure. Transducers in both groups have symmetric conductivity, $\overline{\bar{\sigma}}$, permittivity $\overline{\bar{\epsilon}}$, permeability $\overline{\bar{\mu}}$, Hooke's tensor $\overline{\bar{c}}$; and magneto-electric tensors related by $\overline{\bar{\nu}}=-\overline{\bar{\tau}}_{t}$. Transducers of the first group have, in addition, the piezoelectric and piezomagnetic tensors related by

$$
\begin{aligned}
& \overline{\bar{\alpha}}=\overline{\overline{\bar{\alpha}}}_{t}^{\prime} \\
& \overline{\bar{\beta}}=-\overline{\overline{\bar{\beta}}}_{t}^{\prime} .
\end{aligned}
$$

Transducers of the second group satisfy

$$
\begin{gathered}
\overline{\overline{\bar{\alpha}}}=-\overline{\bar{\alpha}}_{t}^{\prime} \\
\overline{\bar{\beta}}=\overline{\overline{\bar{\beta}}}_{t}^{\prime} .
\end{gathered}
$$

Table 1 shows that the $L$ adjoint to transducers in the first group can be realized by simply reversing the direction of the static magnetic induction $\bar{B}_{0}$ which biases the given transducer. (For 
some gyrotropic media, e.g., ferrites, transposition of the permittivity and permeability tensors is also accomplished by the reversal of the magnetic induction. In fact, the reversal of $\bar{B}_{0}$ to form the $L$ adjoint transducer is analagous to the reversal of the dc magnetic field necessary to obtain purely em reciprocit y relationships for gyrotropic media [24].)

Table 1 also shows that the $L^{-}$adjoint to transducers of the second group can be realized simply by reversing the sign of the static charge distribution $\rho_{e}^{0}$, or equivalently, the static electric field and electric polarization which biases the transducer.

The author is grateful to D. M. Kerns for the original suggestion of the work and for the many helpful discussions throughout its completion.

\section{References and Notes}

[1] Kerns, D. M., Correction of near-field antenna measurements made with an arbitrary but known measuring antenna, Electron. Lett. 6, 346-347 (1970).

[2] Kerns, D. M., New method of gain measurement using two identical antennas, Electron. Lett. 6, 348-349 (1970).

[3] Much of the theory was published originally by D. M. Kerns and E. S. Dayhoff, Theory of diffraction in microwave interferometry, J. Res. Nat. Bur. Stand. (U.S.)., 64B, (Math. and Math. Phys.) l-13 (Jan.-Mar. 1960).

[4] Kerns, D. M. Scattering-matrix description and near-field measurements of electroacoustic transducers, Nat. Bur. Stand. (U.S.), Tech. Note. 651, 40 pages (Mar. 1974).

[5] Adjoint reciprocity relations have been derived for antennas by D. M. Kerns, Plane-wave scattering matrix and generalized reciprocity relations for antennas and scatterers, 1972 unpublished report.

[6] Foldy, L. L., and Primakoff, H., A general theory of passive linear electroacoustic transducers and the electroacoustic reciprocity theorem. I, J. Acoust. Soc. Amer. 17, 109-120 (1945).

[7] Primakoff, H., and Foldy, L. L., A general theory of passive linear electroacoustic transducers and the electroacoustic reciprocity theorem. II, J. Acoust. Soc. Amer. 19,50-58 (1947).

[8] Newell, A. D., Baird, R. C., and Wacker, P. F., Accurate measurement of antenna gain and polarization at reduced distances by an extrapolation technique, IEEE Trans. Antenas Propagat. AP-2 1, 418-431 (1973).

[9] Yaghjian, A. D., Theory of adjoint reciprocity for electroacoustic transducers, National Bureau of Standards, 1974, unpublished report.

[10] McMillan, E. M., Violation of the reciprocity theorem in linear passive electromechanical systems, J. Acoust. Soc. Amer. 18,344-347 (1946). The useful term "antireciprocity" was apparently introduced by McMillan.

[11] Morse, P. M., and Feshbach, H., Methods of Theoretical Physics Part 1, Sect. 1.6 (McGraw-Hill, New York, 1953).

[12| Auld, B. A.. Application of microwave concepts to the theory of acoustic fields and waves in solids. IEEE Trans. Microwave Theory Tech. MTT-1 7, 800-811 (1969).

[13| O’Dell, T. H., The Electrodynamics of Magneto-Electric Media, (Selected Topics in Solid State Physics, E. P. Wohlfarth, Ed., Vol. 11), (North-Holland/American Elsevier, New York, 1970).

[14] Berlincourt, D. A., Curran, D. R., and Jaffe, H., Piezoelectric and Piezomagnetic Materials and their Function in Transducers, in Physical Acoustics, W. P. Mason, Ed. (Academic Press, New York 1964). 1A, Chap. 3

[15] Thurston, R. N., Wave Propagation in Fluids and Normal Solids, in Physical Acoustics, W. P. Mason, Ed., New York (Academic Press, New York 1964), 1A, Chap. 1, pp. 11-18.

[16] Lorrain, P., and Corson, D. R., Electromagnetic Fields and Waves (W. H. Freeman, San Francisco, 1970), 2d ed., Chap. 10, pp. 446-448.

[17] Lanczos, C., Linear Differential Operators (D. Van Nostrand, London, 1961), Chap. 4. See also Ref. 11, Sect. 7.5.

[18] Lewis, J. A., The effect of driving electrode shape on the electrical properties of peizoelectric crystals, Bell Syst. Tech. J. 40, 1259-1280 (1961).

[19] Kerns, D. M., Plane-wave spectra and spectral coupling products for arbitrary radiating and scattering systems, 1922 , unpublished report.

[20] American Standard Acoustical Terminology, New York: American standards association, 1960, S1.1-1960, Sect. 7.30, pp. 32-33.

[21] Morse, P. M., and Ingard, K. U., Theoretical Acoustics. New York: (McGraw-Hill, New York, 1968), Chap. 8, p. 405. Adjoint reciprocity principles for acoustic scatterers derive entirely from a scattering matrix having the symmetry properties of $S_{11}$ (eq $(53 \mathrm{~d})$ ). The analagous symmetry or "reciprocity theorem" exists for the scattered plane wave amplitudes of quantum mechanics (see ref. [11], part 2, Chap. 9, pp. 1130-1131).

[22] Mirsky, L., An Introduction to Linear Algebra, (Oxford, London, 1961), Chap. 13.

[23] Mercer, J., Functions of positive and negative type, and their connection with the theory of integral equations, Proc. Roy. Soc. Ser. A, 83, No. A559,69-70 (1909).

[24] Harrington, R. F., and Villeneuve, A. T., Reciprocity relationships for gyrotropic media, IRE Trans. Microwave Theory Tech. MTT-6, 308-310 (1958).

(Paper 79B1 \&2-417) 